\title{
On the Capacity of Vector Gaussian Interference Channels
}

\author{
Sriram Vishwanath \\ Electrical and Computer Engineering \\ Univ. of Texas at Austin, \\ Austin, TX 78712 \\ e-mail: sriram@ece.utexas.edu
}

\author{
Syed Ali Jafar \\ Electrical Engineering and Comp. Science \\ Univ. of California Irvine, \\ Irvine, CA 92697 \\ e-mail: syed@ece.uci.edu
}

Abstract - The capacity of a vector Gaussian interference channel is investigated. Outer bounds, and where possible, capacity regions of a class of interference channels is characterized. The analysis of single transmit multiple receive antenna (SIMO) Gaussian interference channels with strong interference can be easily seen to be exactly analogous to that of a single transmit single receive antenna system. This paper demonstrates that, in contrast, multiple transmit single receive antenna (MISO) Gaussian interference channels are much harder to characterize. In this paper, the capacity region for a class of MISO interference channels with very strong interference is characterized. Also, the rank of the optimal transmit policy in a MISO Gaussian interference channel is shown to be bounded by the number of users in the system. Finally, outer bounds on the capacity region of the general multiple transmit and receive antenna (MIMO) Gaussian Interference Channels are derived. A new outer bound is obtained, which combines and improves previously known strategies for bounding the capacity of interference channels.

\section{INTRODUCTION}

The discrete memoryless interference channel (abbreviated IFC) is a two transmit, two receive system $\left(\mathcal{X}_{1} \times\right.$ $\left.\mathcal{X}_{2}, p\left(y_{1} \mid x_{1}, x_{2}\right) p\left(y_{2} \mid x_{1}, x_{2}\right), \mathcal{Y}_{1} \times \mathcal{Y}_{2}\right)[1,4]$, as shown in Figure 1. This channel has two independent messages, one at each transmitter $i \in 1,2$, intended for receiver $i$.

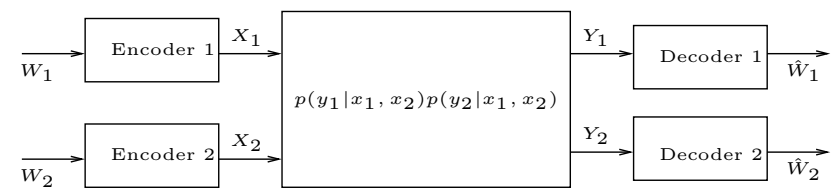

Figure 1: Discrete Memoryless Interference Channel

The scalar Gaussian interference channel is of the form (Figure 2)

$$
\begin{aligned}
& Y_{1}=X_{1}+\sqrt{\alpha} X_{2}+N_{1} \\
& Y_{2}=\sqrt{\beta} X_{1}+X_{2}+N_{2} .
\end{aligned}
$$

with $\alpha, \beta \in \mathbb{R}^{+} . N_{1}, N_{2}$ are Gaussian distributed with zero mean and unit variance. The transmit power for transmitter $i$ is assumed to be $P_{i}, i \in\{1,2\}$.

Although the capacity region of both the discrete memoryless IFC and the particular case of the Gaussian IFC are still open problems, the capacity regions of many families of IFCs satisfying a given set of constraints have been found $[4,9]$.

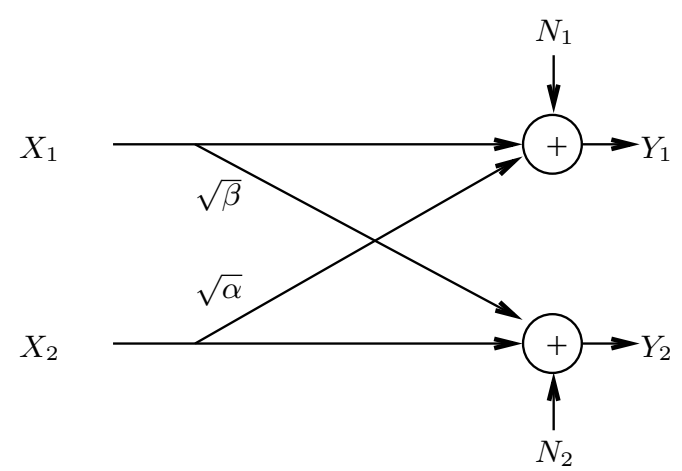

Figure 2: Scalar Gaussian Interference Channel

The first family of IFCs for which the capacity region is known is that of twin interference channels [2]. Subsequently, the capacity region very strong interference channels was characterized in [3]. Very strong IFCs satisfy the constraints

$$
\begin{aligned}
& I\left(X_{1} ; Y_{1} \mid X_{2}\right) \leq I\left(X_{1} ; Y_{2}\right) \\
& I\left(X_{2} ; Y_{2} \mid X_{1}\right) \leq I\left(X_{2} ; Y_{1}\right) .
\end{aligned}
$$

for all $\left(X_{1}, X_{2}\right) \sim p\left(X_{1}\right) p\left(X_{2}\right)$. For the scalar Gaussian IFC (2) reduces to

$$
\begin{aligned}
& \alpha \geq P_{1}+1 \\
& \beta \geq P_{2}+1
\end{aligned}
$$

The authors of [9] found that this very strong interference requirement in Equation (2) can be considerably weakened while still being able to characterize the capacity region. Their approach leads to the strong interference conditions ([9])

$$
\begin{aligned}
& I\left(X_{1} ; Y_{1} \mid X_{2}\right) \leq I\left(X_{1} ; Y_{2} \mid X_{2}\right) \\
& I\left(X_{2} ; Y_{2} \mid X_{1}\right) \leq I\left(X_{2} ; Y_{1} \mid X_{1}\right),
\end{aligned}
$$

for all $p\left(X_{1}\right) p\left(X_{2}\right)$. It is easy to show that (4) is a superset of (2). It is shown in [9] that the capacity region of an interference channel satisfying Equation (4) is given by the intersection of the capacity regions of two multiple-access channels. The equivalent strong interference conditions for scalar Gaussian IFCs were found in [6] to be $\alpha, \beta \geq 1$. Disregarding isolated cases, the strong IFCs form the largest family of IFCs for which the capacity region is known.

To handle non-strong IFCs, substantial contributions have been made in obtaining good outer bounds on the capacity regions of IFCs $[12,13,7,10]$. These outer bounds enable us to gauge the proximity of achievable strategies to the ultimate capacity limit. The outer bounds obtained recently by Kramer [12] are the best known outer bounds for general IFCs today.

This paper has the following two goals. The first is to specialize the strong interference conditions in (4) to the vector 
Gaussian IFC to obtain 1) closed-form expressions for the constraints on the set of strong IFCs in terms of channel parameters, and 2) closed-form expressions for the capacity region of vector Gaussian strong IFCs. In subsequent sections, we shall find that both 1) and 2) are difficult to obtain in many cases, and that alternate constraints have to be derived for those cases.

The second goal of this paper is to derive improved outer bounds on the capacity of Gaussian IFCs based on [12] that are tailored to the vector Gaussian IFC capacity problem. We find that many of the bounding strategies used for scalar Gaussian IFCs are no longer applicable in the vector case, and that alternate techniques need to be applied to obtain good outer bounds.

The paper is organized as follows. The concept of strong interference encoding and decoding for a scalar Gaussian IFC is explained by combining in the next section by combining techniques from [6] and [10]. This notion of strong interference encoding and decoding is generalized to the SIMO Gaussian IFC, and thus the capacity region of a SIMO Gaussian IFC with strong interference is detailed in Section III. A class of MISO Gaussian IFCs were capacity can be characterized in closed form is illustrated in Section IV, and finally, specialized outer bounds for the MIMO Gaussian IFC are presented in Section V. The paper concludes with Section VI.

\section{Scalar Gaussian IFCs}

In this section, we describe the strong interference capacity result for scalar Gaussian IFCs (in Figure 2) by combining the techniques introduced by Sato [6] and Costa [10]. Consider the two interference channels given by

$$
\begin{aligned}
& Y_{1}=X_{1}+N_{1} \\
& Y_{2}=\sqrt{\beta} X_{1}+X_{2}+N_{2}
\end{aligned}
$$

and

$$
\begin{aligned}
& Y_{1}=X_{1}+\sqrt{\alpha} X_{2}+N_{1} \\
& Y_{2}=X_{2}+N_{2} .
\end{aligned}
$$

The capacity regions of both channels (5) and (6) are outer bounds on the capacity region of (1) [10]. Moreover, the capacity region for (5) for $\beta \geq 1$ is bounded above by the capacity region of the multiple-access channel [6]

$$
Y_{2}=\sqrt{\beta} X_{1}+X_{2}+N_{2} .
$$

Similarly, for $\alpha \geq 1$, the capacity region of (6) is bounded above by the capacity region of the multiple access channel

$$
Y_{1}=X_{1}+\sqrt{\alpha} X_{2}+N_{1} .
$$

Thus, an outer bound on the capacity of (1) is the intersection of the capacity regions of the two MACs considered above. Since this outer bound is also achievable [6], we have the desired strong interference result.

Next, we tackle the simplest vector Gaussian IFC, the SIMO channel.

\section{Single Transmit Multiple Receive Systems}

This system is shown in Figure 3 and is given mathematically by

$$
\begin{aligned}
& \mathbf{Y}_{1}=\mathbf{H}_{1} X_{1}+\mathbf{H}_{2} X_{2}+\mathbf{N}_{1} \\
& \mathbf{Y}_{2}=\mathbf{H}_{3} X_{1}+\mathbf{H}_{4} X_{2}+\mathbf{N}_{2}
\end{aligned}
$$

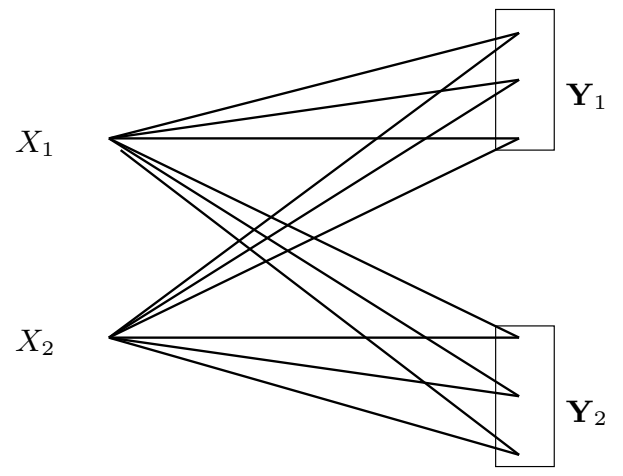

Figure 3: Single Transmit Multiple Receive Interference Channel

In this system, the additive noise vectors $\mathbf{N}_{i}, i=1,2$ are assumed to be zero mean Gaussian random vectors with identity covariance. The channel vectors $\mathbf{H}_{i}, i=1, \ldots, 4$ are assumed to be static and known to the transmitters and the receivers. Without loss of generality we assume that $\left\|\mathbf{H}_{1}\right\|=\left\|\mathbf{H}_{4}\right\|=1$. We seek to obtain relationships amongst the channel parameters $\mathbf{H}_{i}, i=1, \ldots, 4$ such that the capacity region of this SIMO interference channel can be characterized in closed form.

Towards this aim, the combination of the Costa and Sato techniques described in the previous section (Section II) to analyze the scalar Gaussian IFC is applied to the SIMO Gaussian IFC. Thus, a "Z" interference channel of the form

$$
\begin{aligned}
& \mathbf{Y}_{1}=\mathbf{H}_{1} X_{1}+\mathbf{N}_{1} \\
& \mathbf{Y}_{2}=\mathbf{H}_{3} X_{1}+\mathbf{H}_{4} X_{2}+\mathbf{N}_{2}
\end{aligned}
$$

is obtained whose capacity region is an outer bound on the capacity of the original SIMO IFC (Equation (7)).

The next important step is to note that performing an invertible unitary transformation on the outputs $\mathbf{Y}_{i}, i=1,2$ does not change the capacity of the overall system. A smart choice for a unitary transformation on $\mathbf{Y}_{1}$ yields a new channel

$$
\mathbf{Y}_{1}^{\prime}=\left[\left\|\mathbf{H}_{1}\right\|, 0, \cdots, 0\right]^{T} X_{1}+\mathbf{N}_{1}^{\prime},
$$

where $\mathbf{N}_{1}^{\prime}$ is identical in distribution to $\mathbf{N}_{1}$. Similarly $\mathbf{Y}_{2}$ can be losslessly transformed to $\mathbf{Y}_{2}^{\prime}=\left[\left\|\mathbf{H}_{3}\right\|, 0, \cdots, 0\right] X_{1}+$ $\mathbf{H}_{4}^{\prime} X_{2}+\mathbf{N}_{2}^{\prime}$, yielding a new channel

$$
\begin{aligned}
Y_{11}^{\prime} & =\left\|\mathbf{H}_{1}\right\| X_{1}+\mathbf{N}_{1}^{\prime} \\
\mathbf{Y}_{2}^{\prime} & =\left[\left\|\mathbf{H}_{3}\right\|, 0, \cdots, 0\right] X_{1}+\mathbf{H}_{4}^{\prime} X_{2}+\mathbf{N}_{2}^{\prime}
\end{aligned}
$$

The new channel defined by Equation (9) has the same capacity region as that defined by Equation (8). Finally, we use arguments analogous to those employed in [6] for the scalar IFC. If a particular rate pair $\left(R_{1}, R_{2}\right)$ belong to the capacity region of the SIMO Gaussian IFC (Equation (8)), then the receiver corresponding to $\mathbf{Y}_{2}^{\prime}$ by definition of capacity, is required to be able to reconstruct $X_{2}$ with an arbitrarily small error. Hence, $\mathbf{Y}_{2}^{\prime}$ can construct

$$
\mathbf{Y}_{2}^{\prime \prime}=\mathbf{Y}_{2}^{\prime}-\mathbf{H}_{4}^{\prime} X_{2}=\left[\left\|\mathbf{H}_{3}\right\|, \cdots, 0\right] X_{1}+\mathbf{N}_{2}^{\prime}
$$

with an arbitrarily small error. Now, if $\left\|\mathbf{H}_{3}\right\| \geq 1=\left\|\mathbf{H}_{1}\right\|$, then $X_{1}$ can also be decoded with an arbitrarily small error from $\mathbf{Y}_{2}^{\prime \prime}$. 
In other words, in the interference channel defined by (8) with $\left\|\mathbf{H}_{3}\right\| \geq 1$, the receiver corresponding to $Y_{2}$ possesses the ability to decode both $X_{1}$ and $X_{2}$ with arbitrarily small error. Thus the capacity region of (8) and thus that of the MISO Gaussian IFC (7) is bounded above by

$$
\begin{aligned}
R_{1} & \leq \frac{1}{2} \log \left|I+\mathbf{H}_{3} \mathbf{H}_{3}^{T} P_{1}\right| \\
R_{2} & \leq \frac{1}{2} \log \left|I+\mathbf{H}_{4} \mathbf{H}_{4}^{T} P_{2}\right| \\
R_{1}+R_{2} & \leq \frac{1}{2} \log \left|I+\mathbf{H}_{3} \mathbf{H}_{3}^{T} P_{1}+\mathbf{H}_{4} \mathbf{H}_{4}^{T} P_{2}\right|
\end{aligned}
$$

The same argument can be repeated for the "Z" interference channel

$$
\begin{aligned}
Y_{1} & =\mathbf{H}_{1} X_{1}+\mathbf{H}_{2} X_{2}+N_{1} \\
Y_{2} & =\mathbf{H}_{4} X_{2}+N_{2}
\end{aligned}
$$

to obtain a second outer bound on the capacity region of (7) for $\left\|\mathbf{H}_{2}\right\| \geq 1=\left\|\mathbf{H}_{4}\right\|$. It is easy to show that the intersection of the outer bounds given by the two multiple access channels capacity regions is achievable. Thus, we have completely characterized the capacity of (7) for $\left\|\mathbf{H}_{2}\right\|,\left\|\mathbf{H}_{3}\right\| \geq 1$.

Note that the same result can also be obtained by applying the strong interference condition (4) [9]. However, the strategy described above is enhances our intuitive understanding of the problem, and is also constructive.

Another case in which the capacity of this system allows easy characterization is when $\mathbf{H}_{1}^{T} \mathbf{H}_{2}=\mathbf{H}_{2}^{T} \mathbf{H}_{4}=0$, i.e., when the signal and interference are orthogonal. In this case, the system reduces to two parallel channels.

\section{Multiple Transmit Single Receive Systems}

This system is shown in Figure 4 and is given mathematically by

$$
\begin{aligned}
& Y_{1}=\mathbf{H}_{1}^{T} \mathbf{X}_{1}+\mathbf{H}_{2}^{T} \mathbf{X}_{2}+N_{1} \\
& Y_{2}=\mathbf{H}_{3}^{T} \mathbf{X}_{1}+\mathbf{H}_{4}^{T} \mathbf{X}_{2}+N_{2} .
\end{aligned}
$$

where $\mathbf{H}_{i}, i=1, \ldots, 4$ and $\mathbf{X}_{j}, j=1,2$ are $1 \times m$ column vectors, $\left\|\mathbf{H}_{1}\right\|=\left\|\mathbf{H}_{4}\right\|=1$, and the noise at each receiver is assumed to be zero mean Gaussian with unit variance. Unlike

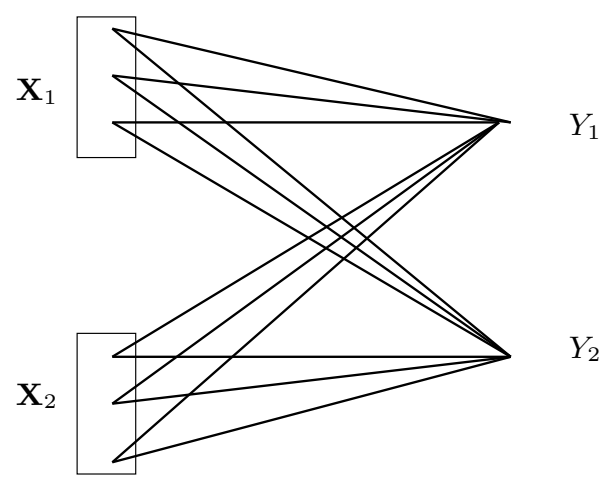

Figure 4: Multiple Transmit Single Receive Interference Channel

the multiple receive case, the capacity region for this channel is considerably more difficult to analyze even in the strong interference case. Take for instance the strong interference condition (4):

$$
I\left(X_{1} ; Y_{1} \mid X_{2}\right) \leq I\left(X_{1} ; Y_{2} \mid X_{2}\right)
$$

which in this case reduces to

$$
h\left(\mathbf{H}_{1}^{T} \mathbf{X}_{1}+\mathbf{N}_{1}\right)-h\left(\mathbf{N}_{1}\right) \leq h\left(\mathbf{H}_{3}^{T} \mathbf{X}_{1}+\mathbf{N}_{2}\right)-h\left(\mathbf{N}_{2}\right)
$$

where $h(\cdot)$ represents the differential entropy function. For the channel to be characterized as a it strong interference channel, this inequality (12) must hold for all input distributions on $\mathbf{X}_{1}$. Unlike the SIMO IFC case, it is very difficult to characterize the family of channels satisfying (12) without imposing specific structural constraints on both $\mathbf{H}_{1}$ and $\mathbf{H}_{2}$. Thus, a direct application of the strong interference conditions (4) for the MISO IFC case does not lead to explicit conditions for all channel realizations.

To present a tractable methodology for identifying IFCs where the channel capacity region can be characterized, we utilize an approach analogous to that for SIMO channels in Section 3 (and scalar channels in Section 2). Consider the "Z" interference channel of the form

$$
\begin{aligned}
& Y_{1}=\mathbf{H}_{1}^{T} \mathbf{X}_{1}+N_{1} \\
& Y_{2}=\mathbf{H}_{3}^{T} \mathbf{X}_{1}+\mathbf{H}_{4}^{T} \mathbf{X}_{2}+N_{2}
\end{aligned}
$$

Now, we perform an invertible unitary transformation $\mathbf{U}_{1}$ on the input $\mathbf{X}_{1}$ to obtain $\mathbf{X}_{1}^{\prime}=\mathbf{U}_{1} \mathbf{X}_{1}$. Note that replacing $\mathbf{X}_{1}$ by $\mathbf{X}_{1}^{\prime}$ as the transmitted signal in (13) does not impact the capacity of the system, and the power constraint on the transmitter $\operatorname{Tr}\left(\mathbf{U} \mathbf{X}_{1}\right)=\operatorname{Tr}\left(\mathbf{X}_{1}\right)$, and is hence satisfied. Thus, a new system that has the same capacity region is

$$
\begin{aligned}
& Y_{1}=\mathbf{H}_{1}^{T} \mathbf{U}_{1} \mathbf{X}_{1}+N_{1} \\
& Y_{2}=\mathbf{H}_{3}^{T} \mathbf{U}_{1} \mathbf{X}_{1}+\mathbf{H}_{4}^{T} \mathbf{X}_{2}+N_{2}
\end{aligned}
$$

Let $\mathbf{X}_{1} \triangleq\left[x_{11}, \ldots, x_{1 m}\right]$. We choose $\mathbf{U}_{1}$ as a unitary matrix with its first column given by the normalized channel vector $\frac{\mathbf{H}_{1}}{\left\|\mathbf{H}_{1}\right\|}=\mathbf{H}_{\mathbf{1}}$. The remaining columns are generated using a Gram-Schmidt orthonormalization procedure on the columns of an identity matrix. This choice of $\mathbf{U}_{1}$ permits us to rewrite the system in (14) as

$$
\begin{aligned}
& Y_{1}=[1,0, \cdots, 0] \mathbf{X}_{1}+N_{1} \\
& Y_{2}=\mathbf{H}_{3}^{T} \mathbf{U}_{1} \mathbf{X}_{1}+\mathbf{H}_{4}^{T} \mathbf{X}_{2}+N_{2},
\end{aligned}
$$

The only component of $\mathbf{X}_{1}$ reaching receiver $Y_{1}$ is $x_{11}$. Intuitively, the only reason for non-zero components $x_{12}$ through $x_{1 m}$ is to "aid" the decoding of $\mathbf{X}_{2}$ at Receiver 2 .

The maximum rate achievable by Transmitter 2 (with corresponding transmit signal $\mathbf{X}_{2}$ is when $\mathbf{X}_{1}$ is absent from the system. Equivalently, when $\mathbf{H}_{3}^{T} \mathbf{H}_{1} \geq 1+P_{2}, Y_{2}$ is guaranteed the ability to decode $\mathbf{X}_{1}$ irrespective of all other parameters in the system, including the transmit policy of $\mathbf{X}_{2}$. Under this assumption, the two channels in (15) decouple, and the capacity achieving strategy in (15) sets $x_{12}, \ldots, x_{1 m}=0$. Hence the system reduces to

$$
\begin{aligned}
Y_{1} & =x_{11}+N_{1} \\
Y_{2} & =\mathbf{H}_{3}^{T} \mathbf{H}_{1} x_{11}+\mathbf{H}_{4}^{T} \mathbf{X}_{2}+N_{2} .
\end{aligned}
$$


The capacity region of (15) is equivalent to that of a system with two parallel non-interfering users, given by

$$
\begin{aligned}
& Y_{1}=x_{11}+N_{1} \\
& Y_{2}=x_{21}+N_{2} .
\end{aligned}
$$

which is clearly an outer bound on capacity. Considering the system

$$
\begin{aligned}
Y_{1} & =\mathbf{H}_{1}^{T} \mathbf{X}_{1}+\mathbf{H}_{2}^{T} \mathbf{X}_{2}+N_{1} \\
Y_{2} & =\mathbf{H}_{4}^{T} \mathbf{X}_{2}+N_{2} .
\end{aligned}
$$

If $\mathbf{H}_{2}^{T} \mathbf{H}_{4} \geq 1+P_{1}$, it can be similarly deduced that the capacity of this system is outer bounded by that of (15).

These outer bounds on capacity are apparently simple ones. It is important to note, however, that they are also tight for the overall MISO IFC (11) when $\mathbf{H}_{2}^{T} \mathbf{H}_{4} \geq 1+P_{1}$ and $\mathbf{H}_{3}^{T} \mathbf{H}_{1} \geq$ $1+P_{2}$. Also, the conditions derived above can be shown to be equivalent to the very strong interference conditions (2). Thus, unlike the strong interference conditions (4), the mutual information based very-strong interference conditions (2) can be translated into closed form channel constraints $\mathbf{H}_{3}^{T} \mathbf{H}_{1} \geq$ $1+P_{2}$ and $\mathbf{H}_{2}^{T} \mathbf{H}_{4} \geq 1+P_{1}$. Moreover, capacity under these constraints can also be characterized in closed form.

The unitary transformations discussed above also lead to the following observation: For the multiple transmit single receive interference channel described by (11), the rank of the input covariance matrix of each transmitter is no more than the number of users. For two users we have,

$$
\operatorname{rank}\left(\mathrm{E}\left[\mathbf{X}_{i} \mathbf{X}_{i}^{T}\right]\right) \leq 2, \quad i \in\{1,2\}
$$

The result of (18) is easily seen by applying two unitary transformations $\mathbf{U}_{1}$ and $\mathbf{U}_{2}$ to the input $\mathbf{X}_{1}$. First, $\mathbf{U}_{1}$ is chosen so that $\mathbf{H}_{1}^{T} \mathbf{U}_{1}$ is reduced to the new channel vector $\mathbf{H}_{1}^{[1]}=[1,0, \cdots, 0]$ as described above. Note that $\mathbf{U}_{1}$ also effectively rotates the channel vector $\mathbf{H}_{3}$ into $\mathbf{H}_{3}^{[1]}=\mathbf{H}_{3}^{T} \mathbf{U}_{1}$. The second unitary transformation $\mathbf{U}_{2}$ is chosen so that the first row and column of $\mathbf{U}_{2}$ are the same as an identity matrix. Thus, $\mathbf{H}_{1}^{T} \mathbf{U}_{1} \mathbf{U}_{2}=\mathbf{H}_{1}^{T} \mathbf{U}_{1}=[1,0, \cdots, 0]$ is not affected by the second transformation. However, the remaining $(m-1) \times(m-1)$ unitary submatrix $\mathbf{U}_{2}^{\prime}$ of $\mathbf{U}_{2}$ is chosen to rotate $\mathbf{H}_{3}^{[1]}$ into $\mathbf{H}_{3}^{[2]}=\mathbf{H}_{3}^{[1]} \mathbf{U}_{2}$ where only the first two elements of $\mathbf{H}_{3}^{[2]}$ can be non-zero. This is achieved by choosing the first column of $\mathbf{U}_{2}^{\prime}$ as the normalized $(m-1) \times 1$ sub-vector of $\mathbf{H}_{3}^{[1]}$ obtained by omitting the first element. Similar rotations can be applied to the input $\mathbf{X}_{2}$ and the resulting channel is shown in Fig. 5. Thus, the unitary transformations applied to the vector inputs eliminate the redundant spatial dimensions and we are left with only as many transmit antennas at each user as the number of users. The result of (18) follows because the rank of the input covariance matrix can not be more than the effective number of dimensions. For a more detailed explanation of these transformations, we refer the reader to [15] where these transformations have been used to eliminate redundant spatial dimensions in the context of vector multiple access and broadcast channels.

\section{Multiple Transmit and Receive Systems: OUTER BOUNDS}

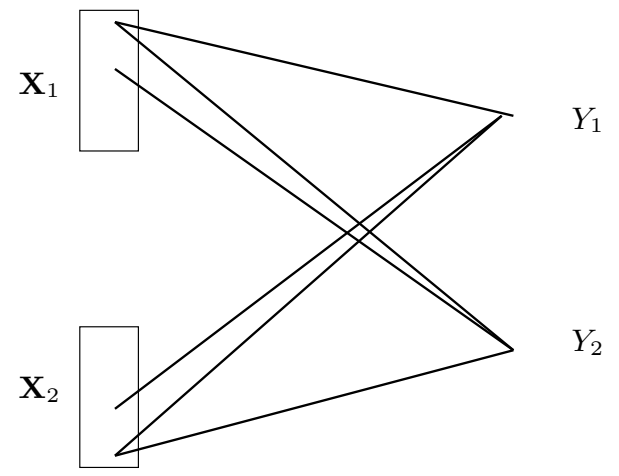

Figure 5: Equivalent Multiple Transmit Single Receive Interference Channel

In the general case where there are both multiple transmit and receive antennas in the system, deriving closed-form expressions for both the strong and the very-strong interference cases becomes a tedious task. Thus, it is very useful to focus on deriving clever outer bounds on capacity, and then to isolate conditions under which the upper bounds are tight, i.e., yield capacity expressions.

Considerable effort has been put into obtaining outer bounds on the capacity region for discrete memoryless IFCs $[12,11,8,10]$. Also, outer bounds specific to subclasses such as the scalar Gaussian IFC have been designed that are amongst the best known outer bounds for these channels [8, 12]. However, the outer bounds obtained for discrete memoryless IFCs are based on principles that are common to all IFCs, and hence are weak outer bounds when applied to MIMO Gaussian IFCs. On the other hand, outer bounds developed for scalar Gaussian IFCs are too specific, and do not always generalize to the MIMO case. In this section, we list the set of applicable outer bounds to the MIMO IFC case, and obtain a new outer bound based on the principles developed in Sections II - IV.

Primarily, two families of outer bounds on the capacity region of the discrete memoryless IFC channel have been studied. The first is that of genie-aided outer bounds, where limited extra information is provided to either one receiver (say $\mathbf{Y}_{1}$ ) or to both receivers simultaneously [12]. A new channel is now generated whose capacity region is a superset of the channel under consideration. The aim of the genie aided outer bound is to render the capacity problem of the new channel tractable, while ensuring that "minimal" information is transferred between receivers. The second approach, pioneered by Sato in [8], is for degraded IFCs, where transmitter cooperation is introduced to generate a degraded broadcast channel. Recent work by Kramer in [12] finds improved bounds for both of these techniques, placing an emphasis on the scalar Gaussian IFC. Interestingly, most of the bounds introduced in [12] are also applicable to the MIMO Gaussian IFC case.

\section{A New Outer Bound}

A new outer bound on the capacity of MIMO IFCs can be obtained by combining Costa's bounding strategy [10] with Sato's receiver cooperation strategy [7]. Consider the MIMO IFC given by: 


$$
\begin{aligned}
& \mathbf{Y}_{1}=\mathbf{H}_{1} \mathbf{X}_{1}+\mathbf{H}_{2} \mathbf{X}_{2}+\mathbf{N}_{1} \\
& \mathbf{Y}_{2}=\mathbf{H}_{3} \mathbf{X}_{1}+\mathbf{H}_{4} \mathbf{X}_{2}+\mathbf{N}_{2} .
\end{aligned}
$$

Using the strategy developed in the previous sections, the capacity of this channel is outer bounded by that of

$$
\begin{aligned}
& \mathbf{Y}_{1}=\mathbf{H}_{1} \mathbf{X}_{1}+\mathbf{N}_{1} \\
& \mathbf{Y}_{2}=\mathbf{H}_{3} \mathbf{X}_{1}+\mathbf{H}_{4} \mathbf{X}_{2}+\mathbf{N}_{2} .
\end{aligned}
$$

Now, we provide $\mathbf{Y}_{1}$ to Receiver 2. This transfer enhances the capacity region of the entire system. Simultaneously, we exploit a result realized by Sato that the capacity region of the IFC is dependent solely on the marginals, and hence "control" the increase in capacity resulting from providing $\mathbf{Y}_{2}$ with added information by allowing the joint distribution between $\mathbf{N}_{1}$ and $\mathbf{N}_{2}$ to be an arbitrary Gaussian distribution with fixed marginals.

Let the covariance for the noise vector $\mathbf{N}=\left[\begin{array}{ll}\mathbf{N}_{1} & \mathbf{N}_{2}\end{array}\right]$ be given by $\mathbf{Z}$. The new channel thus obtained is:

$$
\begin{aligned}
\mathbf{Y}_{1} & =\mathbf{H}_{1} \mathbf{X}_{1}+\mathbf{N}_{1} \\
\mathbf{Y}_{2}^{\prime} & =\mathbf{H}_{3}^{\prime} \mathbf{X}_{1}+\mathbf{H}_{4}^{\prime} \mathbf{X}_{2}+\mathbf{N}
\end{aligned}
$$

where $\mathbf{H}_{3}^{\prime}=\left[\begin{array}{ll}\mathbf{H}_{3} & \mathbf{H}_{1}\end{array}\right]^{T}$ and $\mathbf{H}_{4}^{\prime}=\left[\begin{array}{ll}\mathbf{H}_{4} & \mathbf{0}\end{array}\right]^{T}$. This new Interference channel is degraded as Receiver 2 is capacble of decoding both $\mathbf{X}_{1}$ and $\mathbf{X}_{2}$. Thus, the capacity region of (21) is bounded above by that of the multiple-access channel given by

$$
\mathbf{Y}_{2}^{\prime}=\mathbf{H}_{3}^{\prime} \mathbf{X}_{1}+\mathbf{H}_{4}^{\prime} \mathbf{X}_{2}+\mathbf{N}
$$

The capacity region of the MIMO multiple-access channel is well known, and is given by

$$
\begin{aligned}
& R_{1} \leq \log \left|I+\mathbf{Z}^{-1} \mathbf{H}_{3}^{\prime} \mathbf{S}_{1} \mathbf{H}_{3}^{\prime T}\right| \\
& R_{2} \leq \log \left|I+\mathbf{H}_{4} \mathbf{S}_{2} \mathbf{H}_{4}^{T}\right| \\
& R_{1}+R_{2} \leq \log \left|I+\mathbf{Z}^{-1}\left(\mathbf{H}_{3}^{\prime} \mathbf{S}_{1} \mathbf{H}_{3}^{\prime T}+\mathbf{H}_{4}^{\prime} \mathbf{S}_{2} \mathbf{H}_{4}^{\prime T}\right)\right|
\end{aligned}
$$

A similar outer bound can be obtained by first removing $\mathbf{X}_{1}$ from the $\mathbf{Y}_{2}$ term in (19), and next transferring information from $\mathbf{Y}_{2}$ to $\mathbf{Y}_{1}$. This yields an outer bound of the form

$$
\begin{array}{ll}
R_{1} & \leq \log \left|I+\mathbf{H}_{1} \mathbf{S}_{1} \mathbf{H}_{1}^{T}\right| \\
R_{2} & \leq \log \left|I+\mathbf{W}^{-1} \mathbf{H}_{2}^{\prime} \mathbf{S}_{2} \mathbf{H}_{2}^{\prime T}\right| \\
R_{1}+R_{2} & \leq \log \left|I+\mathbf{W}^{-1}\left(\mathbf{H}_{1}^{\prime} \mathbf{S}_{1} \mathbf{H}_{1}^{\prime T}+\mathbf{H}_{2}^{\prime} \mathbf{S}_{2} \mathbf{H}_{2}^{\prime T}\right)\right|
\end{array}
$$

where $\mathbf{H}_{1}^{\prime}=\left[\begin{array}{ll}\mathbf{H}_{1} & \mathbf{0}\end{array}\right]^{T}, \mathbf{H}_{2}^{\prime}=\left[\begin{array}{ll}\mathbf{H}_{2} & \mathbf{H}_{4}\end{array}\right]^{T}$ and $\mathbf{W}$ the corresponding covariance matrix for $\left[\begin{array}{ll}\mathbf{N}_{1} & \mathbf{N}_{2}\end{array}\right]$. Thus, an intersection of (23) and (24) form a new outer bound on the capacity of (19).

This outer bound is superior to techniques that involve full receiver cooperation (such as the bounds in [7]), as one of the interference terms is absent. For example, in (24), the effect of the $\mathbf{H}_{\mathbf{3}}$ term is removed. The presence of the added interference term can considerably weaken the bound, as it acts as a signal term in the cooperative outer bound.

\section{Conclusions}

This paper addresses the capacity region of the vector interference channel. It investigates the capacity of Single Input Multiple Output (SIMO) interference channels under strong interference conditions, and that of Multiple Input Single Output
(MISO) interference channels under very strong interference. The procedure adopted in this paper to obtain these capacity results is based on simple unitary transformations and intuitive arguments. These techniques can be also used to show that the rank of the optimum transmit policy for the MISO interference channel is no more than the number of users in the system. Finally, outer bounds on the capacity region are detailed, and a new outer bound derived for general Multiple Input Multiple Output (MIMO) Gaussian interference channels.

\section{REFERENCES}

[1] R. Ahlswede, "Multi-way Communication Channels", Second Intl. Symp. Inform. Theory, B. N. Petror and F. Csaki, Eds. Budapest: Akademiai Kiado, 1973, pp. 23-52.

[2] R. Ahslwede, "The Capacity Region of a Channel with Two Senders and Two Receivers", Ann. Prob., vol. 2, pp. 805-814, Oct. 1974

[3] A. B. Carliel, "A Case where Interference Does not Reduce Capacity (Corresp.), IEEE Trans. Inform. Theory, vol. IT-21, pp. 569-570, Sept. 1975.

[4] E. C. van der Meulen, "A Survey of Multi-Way Channels in Information Theory: 1961-1976", IEEE Trans. Inform. Theory, vol. IT-23, pp. 1-37, Jan. 1977.

[5] H. Sato, "On the Capacity of a Discrete Two-user Channel for Strong Interference (Corresp.)", IEEE Trans. Inform. Theory, vol. IT-25, pp. 228-231, Mar. 1979.

[6] H. Sato, "The Capacity of Gaussian Interference Channel under Strong Interference (Corresp.)", IEEE Trans. Inform. Theory, vol. IT-27, pp. 786-788, Nov. 1981.

[7] H. Sato, "Two User Communication Channels", IEEE Trans. Inform. Theory, vol. IT-23, pp. 295-304, Nov. 1985.

[8] H. Sato, "On degraded Gaussian two-user Channels", IEEE Trans. Inform. Theory, vol. IT-24, pp. 637-640, Sep. 1978.

[9] M. H. M. Costa, A. A. El Gamal, "The Capacity Region of the Discrete Memoryless Interference Channel with Strong Interference (Corresp.)", IEEE Trans. Inform. Theory, vol. IT-33, pp.710-711, Sept. 1987.

[10] M. H. M. Costa, "On the Gaussian Interference Channel", IEEE Trans. Inform. Theory, vol. IT-31, pp. 607-615, Sept. 1985.

[11] A. B. Carliel, "Outer Bounds on the Capacity of Interference Channels", IEEE Trans. Inform. Theory, vol. IT-29, pp. 602606, Jul. 1983.

[12] G. Kramer," Outer Bounds on the Capacity of Gaussian Interference Channels", IEEE Trans. Inform. Theory, vol. 50, pp. 581-586, Mar. 2004.

[13] G. Kramer," Genie-aided Outer Bounds on the Capacity of Interference Channels",Proc. IEEE Intern. Symp. Inform. Theory, 2001, pp. 103, 24-29 Jun. 2001.

[14] S. Vishwanath, N. Jindal and A. Goldsmith, "Duality, Achievable Rates and Sum Capacity of Multiple-Antenna Broadcast Channels", IEEE Trans. Inform. Theory, 2003.

[15] S. Jafar and A. Goldsmith, "On the capacity of vector Gaussian MAC and BC with feedback", Proceedings of 41st Annual Allerton Conference on Communication, Control and Computing, October 2003. 